\title{
Enxertos arteriais na cirurgia de revascularização do miocárdio: papel da artéria radial
}

\author{
Armando Bocchi BARLEM*, Eduardo Keller SAADI*, Marcelo Curcio GIB* \\ Waldomiro Carlos MANFROI**
}

RBCCV 44205-530

Barlem A B, Saadi E K, Gib M C, Manfroi W C - Enxertos arteriais na cirurgia de revascularização do miocárdio: papel da artéria radial. Rev Bras Cir Cardiovasc 2001; 16(1): 53-7.

RESUMO: Devido aos baixos índices de permeabilidade dos enxertos de veia safena, a utilização de artérias como enxerto para revascularizar o miocárdio vem sendo muito testada e tem ganho espaço. A revascularização da descendente anterior com a artéria torácica interna esquerda é padrão-ouro neste procedimento e é realizada rotineiramente na maioria dos Serviços do mundo há pelo menos 15 anos. Além disso, existem evidências de que o acréscimo de outro enxerto arterial ao tratamento pode diminuir a morbi-mortalidade tanto intra-hospitalar quanto a longo prazo (10 anos). Na escolha do $2^{\circ}$ enxerto, a artéria radial tem se destacado com bons índices de permeabilidade a médio prazo (84-92\% comparada com safena: $70-80 \%$ em 5 anos). O vasoespasmo, fenômeno inerente aos condutos arteriais, ocorre em 5 a $10 \%$ dos enxertos de radial. A nitroglicerina e os nitratos são as drogas mais eficazes no seu controle e os bloqueadores do cálcio não têm benefício clínico comprovado. Na avaliação da circulação do arco palmar para prevenção de isquemia do membro operado, o Teste de Allen tem sido satisfatório. Parece razoável, portanto, sugerir a associação de outro enxerto arterial à artéria torácica esquerda na tentativa de melhorar os resultados da CRM e que este enxerto seja a artéria radial.

DESCRITORES: Revascularização miocárdica, métodos. Artérias mamárias, transplante. Artéria radial, transplante. Veia safena, transplante.

\section{INTRODUÇÃO}

A veia safena foi a grande responsável pelo desenvolvimento da cirurgia de revascularização do miocárdio (CRM) (1) sendo, ainda hoje, o enxerto de escolha na doença coronariana multiarterial. Todavia, sua baixa patência a médio e longo prazo compromete o resultado cirúrgico final (2-4) levando os cirurgiões a pensarem alternativas para solução deste problema. A utilização de enxertos arteriais na CRM iniciou há 30 anos com a artéria torácica interna esquerda, usualmente chamada de artéria torácica interna esquerda (ATIE) (5) que atingiu ótimos resultados a longo prazo ${ }^{(3,6-8)}$ e atualmente é considerada padrão ouro neste procedimento ${ }^{(9-13)}$. A partir dos benefícios demonstrados com a ATIE, outros enxertos arteriais foram testados com resultados animadores. A vantagem teórica das artérias parece ser o fato de se adaptarem melhor do que a safena às condições hemodinâmicas de aumento de fluxo e pressão sangüínea a que são expostos após a cirurgia. Estas alterações causam na veia hiperplasia intimal precoce levando a posterior aterosclerose e oclusão dos enxertos $3,4,6$. Além disso, enxertos

Trabalho realizado nos Serviços de Cirurgia Cardiovascular do Hospital de Clínicas de Porto Alegre e Serviço de Cardiologia do Hospital de Clínicas de Porto Alegre. RS, Brasil.

Recebido para publicação em novembro de 2000.

* Do Serviço de Cirurgia Cardiovascular do Hospital de Clínicas de Porto Alegre.

** Do Serviço de Cardiologia do Hospital de Clínicas de Porto Alegre.

Endereço para correspondência: Armando B. Barlem. Rua Ramiro Barcellos, 2350 - 2 andar - sala 2060. Porto Alegre, RS, Brasil. CEP 90035-003. Tels. (051) 316-8344. (051) 9977-9338. e-mails: abarlem@zaz.com.br ou esaadi@zaz.com.br 
arteriais são avalvulados e possuem diâmetro mais compatível com a coronária. Tais propriedades conferem aos condutos arteriais uma potencial superioridade de patência em relação à safena. Muitas artérias têm sido propostas como enxertos mas não existe consenso quanto à mais adequada e ao riscobenefício de sua utilização rotineira na CRM. A artéria radial tem sido muito utilizada e tem se destacado pela boa patência, além de fácil obtenção e manipulação (14-16). Alguns autores têm defendido sua utilização associada à ATIE e em substituição à safena, realizando, assim, a revascularização somente com enxertos arteriais, técnica conhecida como "revascularização arterial total" $(14,15,17)$. O objetivo deste trabalho é apresentar uma revisão atualizada das evidências do benefício da utilização de enxertos arteriais na cirurgia de revascularização do miocárdio com ênfase na artéria radial e manejo das suas potenciais complicações.

\section{Benefício Clínico dos Enxertos Arteriais}

Em 1986, LOOP e seus colegas da Cleveland Clinic publicaram uma grande coorte de 10 anos de seguimento de pacientes submetidos a CRM separados em dois grupos: o primeiro com implante de ATIE para a artéria coronária interventricular anterior ou descendente anterior (DA) isoladamente ou em combinação com um ou mais enxertos de safena ( $n=2306)$ e o segundo exclusivamente com enxertos de veia safena ( $n=3625)$. A comparação entre os grupos mostrou aumento da mortalidade e eventos cardíacos nos pacientes que receberam somente safena $(p<0,0001)^{(8)}$ com os seguintes riscos relativos: mortalidade - 1,61, infarto agudo do miocárdio (IAM) - 1,41, hospitalização por eventos cardíacos - 1,25, reoperação - 2,00, e qualquer evento cardíaco - 1,27.

Num estudo semelhante, CAMERON et al.(11) demonstraram em 1996 uma maior sobrevida em 15 anos para pacientes revascularizados com ATIE em comparação com veia safena exclusivamente (risco relativo de morte de 0,73, IC 95\% 0.64-0,83).

Mais recentemente, foi demonstrado que 0 acréscimo de um segundo enxerto arterial ao tratamento também é benéfico.

PICK et al. ${ }^{(12)}$ demonstraram que pacientes que recebem AMI bilateral com ou sem safena têm melhor evolução em 10 anos que os tratados somente com ATIE e safena. A mortalidade no grupo de AMI bilateral foi $15 \%$ e no grupo de ATIE + safena foi $24 \%$ $(p=0,05)$. Houve redução também quanto à ocorrência de IAM $(p=0,19)$ ou qualquer evento cardíaco $(p<0,001)$ e recorrência de angina $(p<0,001)$.

BORGER et al. ${ }^{(18)}$ compararam a evolução intra-hospitalar de pacientes revascularizados com
ATIE + safena e outros com ATIE + um segundo enxerto arterial + safena. Este segundo enxerto arterial era a AMI direita ou artéria radial. Pacientes que receberam dois enxertos arteriais tiveram menor incidência de síndrome de baixo débito cardíaco pós-operatória $(5,6 \%$ vs. $12,0 \%, \mathrm{p}<0,001)$ e IAM peri-operatório $(1,3 \%$ vs. $3,7 \%, p<0,018)$. Não houve diferença estatisticamente significativa entre os grupos quanto a mortalidade $(0,5 \%$ vs. $1,5 \%)$, mas a análise de regressão logística multivariável revelou que a utilização de somente um enxerto arterial (contra dois) foi um forte preditor de mortalidade e morbidade cardíaca (odds ratio=2,2, IC 95\% 1,43,3). Em um segundo tempo o grupo de enxertos arteriais foi comparado internamente sendo que os pacientes que receberam artéria radial ao invés de AMI direita apresentaram menor índice de mediastinite $(0,6 \%$ vs. $5,3 \%, p=0,01)$ e uma tendência a menor necessidade de transfusão sangüínea (40\% vs. $51 \%, p=0,06)$.

BRODMAN et al. ${ }^{(14)}$ realizaram revascularização arterial total em 108 pacientes utilizando ATIE + artéria radial uni ou bilateral e compararam com outros 67 pacientes do mesmo período que receberam revascularização mista (enxertos arteriais + safena). O grupo RAT teve tempo de circulação extracorpórea significativamente reduzido $(84,35 \pm 27,59 \mathrm{~min}$ vs. $109,57 \pm 40,38 \mathrm{~min})$, o mesmo acontecendo com o tempo de clampeamento aórtico $(52,94 \pm 22,79 \mathrm{~min}$ vs. $66,32 \pm 27,46 \mathrm{~min})$. Os autores ainda descrevem que os pacientes do grupo arterial apresentaram menos dor e deambulação mais fácil e precoce do que os safenectomizados, além de um período de internação média de 7 contra 8 dias.

\section{Patência dos Enxertos}

A durabilidade dos enxertos de veia safena e ATIE vem sendo estudada há vários anos e está muito bem documentada. Mais ou menos $10 \%$ dos enxertos de safena podem apresentar oclusão precoce (até 1 ano após a cirurgia) que se deve a qualidade da veia em si e da sua retirada, da coronária recipiente e da anastomose realizada. Numa evolução mais tardia, tem início o processo de hiperplasia intimal e aterosclerose que produz oclusão de $2 \%$ dos enxertos por ano até 5 anos de pós-operatório quando ocorre a fase crítica da aterosclerose que eleva o índice para 5\% por ano até que aos 10 anos de pós-operatório apenas $50 \%$ dos enxertos estão patentes ${ }^{(4,13)}$. A superioridade da ATIE sobre a veia safena em termos de durabilidade é muito grande e a explicação para isto extrapola a simples comparação das propriedades já comentadas de artérias e veias como enxertos. Além das vantagens de conduto arterial, o procedimento padrão para a ATIE é o seu uso como enxerto pediculado para a DA, fato que por si só lhe confere um potencial aumento de patência 
Barlem A B, Saadi E K, Gib M C, Manfroi W C - Enxertos arteriais na cirurgia de revascularização do miocárdio: papel da artéria radial. Rev Bras Cir Cardiovasc 2001; 16(1) 53-7.

devido ao ótimo fluxo possibilitado pelo calibre da DA e seus ramos diagonais e septais. A patência da ATIE nestas condições é cerca de $90 \%$ em 10 anos ou mais $(10,13)$. A revascularização de qualquer outra coronária pela ATIE mesmo pediculada diminui a durabilidade deste enxerto atingindo índices não muito distantes da veia safena ${ }^{(19)}$. Por outro lado, a patência de um enxerto livre de ATIE para a DA é de aproximadamente $80 \%$ em 10 anos $(12,20)$, portanto, inferior a do enxerto pediculado ou in situ.

Quanto à radial, existem muitos trabalhos estudando patência a curto prazo que se mostrou muito similar à da ATIE (cerca de $95 \%$ em até 1 ano) $(14,16,17,21-26)$. Apenas dois outros estudos relatam patência mais tardia. ACAR et al. (27) encontraram um índice de $83 \%$ de sobrevida em 5 anos para enxertos de radial implantados da aorta para vasos do sistema coronariano esquerdo que não a DA. POSSATI et al. (28) relataram $91,9 \%$ de patência em 5 anos de pós-operatório para enxertos de radial também implantados proximalmente à aorta.

\section{Manejo do Vasoespasmo}

Os enxertos arteriais apresentam um potencial risco de espasmo de parede por possuírem uma camada muscular proeminente, o que pode comprometer o resultado cirúrgico imediato. A artéria radial é especialmente suscetível a este fenômeno devido à grande musculatura lisa de sua parede, maior do que a da ATIE. A serotonina, um potente vasoconstritor, induz espasmo in vivo na radial mas não na ATIE (29), o que demonstra a diferente atuação das camadas musculares dos dois vasos. A incidência de vasoespasmo em enxertos de artéria radial gira em torno de 5 a $10 \%(16,30)$. Os bloqueadores do cálcio, liderados pelo diltiazen, foram e ainda são as drogas empiricamente preconizadas para o controle do vasoespasmo no pós-operatório (14-16,18,2123) enquanto que no preparo trans-operatório a maioria dos Serviços utiliza a dilatação intraluminal com papaverina. Apesar da larga utilização, os trabalhos que estudaram a ação dos bloqueadores do cálcio no controle do vasoespasmo in vivo não mostraram benefício ${ }^{(29,30)}$. Em modelos experimentais para produção de espasmo em segmentos de radiais a partir de noradrenalina e $\mathrm{KCl}$, diltiazen e verapamil não preveniriam o fenômeno enquanto que nifedipina foi eficaz contra a noradrenalina. Nitroglicerina e dinitrato de isossorbida foram eficazes contra as duas drogas indutoras. Na reversão do fenômeno, somente nifedipina, dinitrato de isossorbida e nitroglicerina foram eficazes, esta última sendo a mais potente (31).

DALLAN et al. (32) demonstraram bons resultados com uma nova técnica que objetiva a eliminação do vasoespasmo de radial através de um dispositivo que dá suporte externo ao conduto e que originalmente foi desenvolvido para moldar veias de formato não adequado.

Existe unanimidade entre os autores de que uma técnica de dissecção correta com pouco manuseio, evitando traumatizar os enxertos, é de suma importância na prevenção do espasmo.

\section{Avaliação Pré-Operatória do Sistema Arterial Radial-ulnar}

A artéria radial, juntamente com a ulnar, são as artérias responsáveis pela irrigação da mão, existindo, assim, um risco de complicação isquêmica se o enxerto for retirado sem avaliação prévia. Sabe-se que, na maioria das vezes, a ulnar é a dominante e em apenas alguns poucos casos $( \pm 15 \%)$ a radial não pode ser retirada. Vários métodos foram descritos para avaliação deste sistema arterial incluindo medida de fluxo por doppler, cálculo de índice de perfusão por oxímetro, ecodoppler colorido com medida de fluxo e teste de esvaziamento digital da artéria após incisão na pele ${ }^{(14,15,17,23)}$. Apesar disso, muitos outros autores têm utilizado apenas o Teste de Allen como método de avaliação pré-operatória sem registrar nenhuma complicação isquêmica de mão $(18,21,22,27)$.

\section{CONCLUSÃO}

A partir das evidências apresentadas na literatura, parece razoável sugerir que, além de utilizarmos a ATIE para revascularizar a DA em todos os casos possíveis, possamos pensar em acrescentar outro enxerto arterial ao tratamento, com vistas à atingir benefício extra nos pacientes submetidos a revascularização cirúrgica do miocárdio. A artéria radial parece uma ótima opção como segundo enxerto arterial apresentando patência em 5 anos superior à da veia safena e não muito inferior à ATIE, além de ter demonstrado morbidade inferior à da AMI direita. Mais ainda, foi adequada para a realização da revascularização arterial total, técnica que pode, com o crescimento de estudos relacionados ao assunto, confirmar a tendência a menor morbidade do que a técnica convencional. Quanto ao controle do vasoespasmo, parece lógico a manutenção das consagradas medidas de manejo transoperatório com papaverina e, no período de maior risco pós-operatório (primeiras 24h), a infusão contínua de nitroglicerina. Ainda faltam comprovações científicas do valor do tratamento profilático destes pacientes com qualquer vasodilatador via oral. Para avaliação pré-operatória do sistema radial-ulnar, parece definitivo que o Teste de Allen possa ser utilizado como método único. 
Barlem A B, Saadi E K, Gib M C, Manfroi W C - Arterial grafts in CABG: the role of radial artery. Rev Bras Cir Cardiovasc 2001; 16(1):53-7.

ABSTRACT: Because of the low patency rate of saphenous vein grafts, the use of arterial grafts for myocardial revascularization has been increased. The revascularization of the left anterior descending coronary (LAD) with the left internal thoracic artery (ITA) is the gold standard in almost all centers in the last 15 years. There are also evidences that one additional arterial graft decrease short and long-term (10 years) morbi-mortality. Radial artery is an excellent choice for the second arterial graft because of good midterm patency $(84-92 \%$ vs $70-80 \%$ of saphenous vein in 5 years). Spasm, a potential problem in arterial grafts, occurs in 5 to $10 \%$ of radial grafts. Nitroglicerin and nitrates are the most effective drugs in its control and calcium antagonists did not show any clinical benefit. The Allen test alone has been effective to assess patency of collateral blood flow and palmar arch continuity. It seems reasonable, therefore, to suggest association of another arterial graft with left ITA in attempting to improve the results of CABG and that this other graft be the radial artery.

DESCRIPTORS: Myocardial revascularization, methods. Mammary arteries, transplantation. Radial artery, transplantation. Saphenous vein, transplantation.

\section{REFERÊNCIAS BIBLIOGRÁFICAS}

1 Favaloro R G, Effler D B, Groves L K, Sheldon W C, Sones Jr. F M - Direct myocardial revascularization by saphenous vein graft: present operative technique and indications. Ann Thorac Surg 1970;10: 97-111.

2 Geha A S, Krone R J, McCormick J R, Baue A E - Selection of coronary bypass. J Thorac Cardiovasc Surg 1975; 70: 414-31.

3 Campeau L, Enjalbert M, Lespérance J, Vaislic C, Grondin C M, Bourassa M G - Atherosclerosis and late closure of aortocoronary saphenous vein grafts: sequential angiographic studies at 2 weeks, 1 year, 5 to 7 years, and 10 to 12 years after surgery. Circulation 1983; 68: $1-7$.

4 Bourassa M G, Fisher L D, Campeau L, Gillespie M J, McConney $M$, Lespérance $\mathrm{J}$ - Long-term fate of bypass grafts: the Coronary Artery Surgery Study (CASS) and Montreal Heart Institute experiences. Circulation 1985; 72: 71-7.

5 Green G E, Spencer F C, Tice D A, Stertzer S H - Arterial and venous microsurgical bypass grafts for coronary artery disease. J Thorac Cardiovasc Surg 1970; 60: 491-503.

6 Grondin C M, Campeau L, Lespérance J, Enjalbert M, Bourassa M G - Comparison of late changes in internal mammary artery and saphenous vein grafts in two consecutive series of 10 years after operation. Circulation 1984; 70: 208-12.

7 Barner H B, Standeven J W, Reese J - Twelve year experience with internal mammary artery for coronary artery bypass. $J$ Thorac Cardiovasc Surg 1985; 90: 668-75.
8 Loop F D, Lytle B, Cosgrove D M et al. - Influence of the internal-mammary-artery graft on 10-year survival and other cardiac events. $N$ Engl J Med 1986, 314: 1-6.

9 Galbut D L, Traad E A, Dorman M J et al. - Twelve-year experience with bilateral internal mammary artery grafts. Ann Thorac Surg 1985; 40: 264-70.

10 Barner H B \& Barnett M G - Fifteen-to twenty-one-year angiographic assessment of internal thoracic artery as a bypass conduit. Ann Thorac Surg 1994; 57: 1526-8.

11 Cameron A, Davis K B, Green G, Schaff H V - Coronary bypass surgery with internal-thoracic-artery grafts : effects on survival over a 15 -year period. $N$ Engl $J$ Med 1996; 334: 216-9.

12 Pick A W, Orszulak T A, Anderson B J, Schaff H V Single versus bilateral internal mammary artery grafts: 10-year outcome analysis. Ann Thorac Surg 1997; 64: 599-605.

13 Lytle B W, Loop F D, Cosgrove D M, Ratliff N B, Easley $\mathrm{K}$, Taylor $\mathrm{P} \mathrm{C}$ - Long-term (5 to 12 year) serial studies of internal mammary artery and saphenous vein coronary bypass grafts. J Thorac Cardiovasc Surg 1985; 89: 248-58.

14 Brodman R F, Frame R, Camacho M, Chen A, Hollinger I - Routine use of unilateral and bilateral radial arteries for coronary artery bypass graft surgery. J Am Coll Cardiol 1996; 28: 959-63.

15 Weinschelbaum E E, Gabe E D, Macchia A, Smimmo R, Suárez L D - Total myocardial revascularization with internal thoracic arteries. J Thorac Cardiovasc Surg 1997; 114: 911-6. 
Barlem A B, Saadi E K, Gib M C, Manfroi W C - Enxertos arteriais na cirurgia de revascularização do miocárdio: papel da artéria radial. Rev Bras Cir Cardiovasc 2001; 16(1) 53-7.

16 Acar C, Jebara V A, Portoghese M et al. - Revival of the radial artery for coronary artery bypass grafting. Ann Thorac Surg 1992; 54: 652-60.

17 Buxton B F, Fuller J A, Tatoulis J - Evolution of complete arterial grafting. Tex Heart Inst $J$ 1998; 25: 17-23.

18 Borger M A, Cohen G, Buth K J et al. - Multiple arterial grafts: radial versus right internal thoracic arteries. Circulation 1998; 98: 7-14.

19 Huddleston C B, Stoney W S, Alford W C et al. Internal mammary artery grafts: technical factors influencing patency. Ann Thorac Surg 1986; 42: 543.

20 Loop F D, Lytle B W, Cosgrove D M, Golding L A R, Taylor P C, Stewart R W - Free (aorta-coronary) internal mammary artery graft: late results. $J$ Thorac Cardiovasc Surg 1986; 92: 827.

21 Calafiore A M, Teodori G, Giammarco G D et al. Coronary revascularization with the radial artery: new interest for an old conduit. J Card Surg 199; 50: $140-6$.

22 Dietl C A \& Benoit C H - Radial artery graft for coronary revascularization: technical considerations. Ann Thorac Surg 1995; 60: 102-10.

23 Affonso da Costa F D, Affonso da Costa I, Poffo R et al. - Myocardial revascularization with the radial artery: a clinical and angiographic study. Ann Thorac Surg 1996; 62: 475-80.

Chen A H, Nakao T, Brodman R F et al. - Early postoperative angiographic assessment of radial artery grafts used for coronary bypass grafting. $J$ Thorac Cardiovasc Surg 1996; 1208-12.
25 Bhan A, Gupta V, Choudhary S K et al. - Radial artery in CABG: could the early results be comparable to internal mammary artery graft? Ann Thorac Surg 1999; 67: $1631-6$

26 Weinschelbaum E E, Macchia A, Caramutti V M et al. Coronary revascularization surgery with arterial conduits: the technique, results and 4-year follow-up in 1023 consecutive patients. Rev Esp Cardiol 2000; 53: $179-88$.

27 Acar C, Ramsheyi A, Pagny J Y et al. - The radial artery for coronary artery bypass grafting: clinical and angiographic results at five years. J Thorac Cardiovasc Surg 1998; 116: 981-9.

28 Possati G, Gaudino M, Santarelli F et al. - The radial artery in coronary surgery: the midterm clinical and angiographic results and the variation in vasoreactivity over time. G Ital Cardiol 1999; 29: 135-42.

29 Sperti G, Manasse E, Kol A et al. - Comparison of response to serotonin of radial artery grafts and mammary grafts to native coronary arteries and the effect of Diltiazem. Am J Cardiol 1999; 83: 592-6.

30 Arena $\mathrm{G} \&$ Abbate $\mathrm{M}$ - Is calcium antagonist administration necessary after aortocoronary-bypass with the radial artery? Ital Heart J 2000; 256-8.

31 Cable D G, Caccitolo J A, Pearson P J et al. - New approaches to prevention and treatment of radial artery graft vasospasm. Circulation 1998; 98: 15-22.

32 Dallan $L A$, Oliveira $S A$, Figueiredo $L P$, Lisboa $L A$, Platania $F$, Jatene A D - Externally supported radial artery graft for myocardial revascularization: a new technique to avoid vasospasm. J Thorac Cardiovasc Surg 1999; 118: 563-5. 